

\section{EVALUACIÓN DE IMPACTO AMBIENTAL MEDIANTE LA INTRODUCCIÓN DE INDICADORES A UN MODELO BIM DE VIVIENDA SOCIAL}

\section{ENVIRONMENTAL IMPACT ASSESMENT BY MEANS OF INDICATORS EMBEDDED IN A BIM MODEL OF SOCIAL HOUSING}

\author{
MARÍA DEL PILAR MERCADER MOYANO \\ Doctora en Arquitectura \\ Docente e Investigadora Departamento de Construcciones \\ Universidad de Sevilla, Sevilla, España \\ http://orcid.org/0000-0003-2180-4887 \\ pmm@us.es
}

\author{
PATRICIA EDITH CAMPOREALE \\ Doctora en Arquitectura \\ Docente de Posgrado \\ Universidad Nacional de la Plata, Buenos Aires, Argentina \\ https://orcid.org/0000-0001-5808-3230 \\ pcamporeale@fau.unlp.edu.ar
}

ELÍAS CÓZAR-CÓZAR

Doctor en Ingeniería de Edificación

Docente de Posgrado

Universidad de Sevilla, Sevilla, España

https://orcid.org/0000-0002-4053-3421

cozarelias@gmail.com

\section{RESUMEN}

Building Information Modeling (BIM) constituye una herramienta versátil para analizar el ciclo de vida de los edificios y el uso eficiente de los recursos, reducción, reuso y reciclado de residuos de construcción y demolición hacia un parque edilicio sin carbono para 2020 en Europa. Este trabajo propone una nueva metodología para incorporar indicadores: energía incorporada, emisiones de CO2, residuos de construcción y demolición además de sólidos urbanos, en modelos BIM que evalúan el impacto ambiental siguiendo la estructura de la Base de Costes de la Construcción de Andalucía (BCCA). Se eligió un bloque de vivienda social en Sevilla, como caso de estudio, para focalizarse en el análisis de su estructura de hormigón armado debido a su alto impacto.Los resultados agrupados por tarea e indicador muestran el rol preponderante del hormigón. Por el contrario, el acero demuestra ser menos energo-consumidor, generando menos emisiones y permitiendo reducir los residuos mediante reciclaje. Según la discriminación por tareas, se destaca el bajo impacto de las fundaciones frente a la superestructura resistente. Asimismo, cada metro cuadrado de construcción típica genera 76.11 de residuo de construcción y demolición, siendo $76,11 \mathrm{~kg}$ mixto proveniente del hormigón y 0,34kg, del acero.

\section{Palabras clave}

Energía incorporada, emisiones de CO2, residuos de demolición y construcción, diseño por ordenador

\section{ABSTRACT}

Building Information Modeling (BIM) has become a versatile tool to analyze the building life cycle and to achieve the efficient use of natural resources and the reduction, reuse and recycling of construction and demolition waste towards the goal of a decarbonized building stock for 2020 in Europe. This research proposes a new methodology to introduce embodied energy (EE), carbon emissions (CE), construction and demolition waste (CDW), and urban solid waste (USW) indicators into BIM models that assess environmental impact following the structure of the Andalusian Construction

Cost Database (ACCD). A block of social housing was chosen as a case study in order to focus on the analysis of its reinforced concrete structure due to its high impact. The results, grouped by task and indicator, show the dominant role that concrete plays in environmental impact. In contrast, steel proved to consume less energy and generate fewer CE as well. Moreover, steel may be recycled, while reducing the quantity of waste. When analyzed by task, the foundations cause much less impact than the tough superstructure. Likewise, each square meter of the typical housing block generates $76.11 \mathrm{~kg}$ of CDW, with $76.77 \mathrm{~kg}$ of mixed concrete waste and $0.34 \mathrm{~kg}$ of steel waste. 


\section{INTRODUCCIÓN}

El sector de Arquitectura, Ingeniería y Construcción (AEC, por su sigla en inglés) juega un papel clave en el impacto ambiental que provoca la actividad humana. Los edificios son responsables del $40 \%$ del consumo de energía, el $36 \%$ de las emisiones de $\mathrm{CO}_{2}$ y el $40 \%$ de CDW (por su sigla en inglés) en la Unión Europea (UE) ("Edificios Energía - Comisión Europea" 2017). Las directrices de la UE sobre la reducción del consumo de energía (Diario Oficial de la Unión Europea 2010) y la eficiencia energética en los edificios (Diario Oficial de la Unión Europea 2012) determinan que para 2020 cada nuevo edificio estará casi en cero energía (nZEB). Entre los objetivos principales del programa Horizonte 2020 de la UE, el logro de una sociedad y una economía más eficientes en relación con el uso de los recursos naturales y las materias primas y un uso sostenible de la energía aparece como un objetivo principal para luchar contra el cambio climático ("Convención Marco de las Naciones Unidas sobre cambio climático" 2018).

El sector AEC consume más del $20 \%$ del combustible (Tiwari 2001) y el $40 \%$ de los recursos materiales (LópezMesa et al. 2009) en todo el mundo. Este sector enfrenta el desafío de reducir el consumo de energía y CE (por su sigla en inglés), pero el aumento del stock de edificios hace que sea difícil de alcanzar a mediano plazo (Sandberg y Brattebø, 2012).

Años antes, la energía operativa $(\mathrm{OE}$, por su sigla en inglés) representaba el factor principal en el consumo de energía del edificio, mientras que la energía incorporada ( $E E$, por su sigla en inglés) representaba el $10-20 \%$ de la energía en el ciclo de vida del edificio. Sin embargo, a medida que la eficiencia energética disminuye el consumo de $\mathrm{OE}, \mathrm{EE}$ se vuelve más importante para la evaluación del impacto ambiental. González y Navarro consideran que las emisiones de $\mathrm{CO}_{2}$ pueden reducirse al $30 \%$ de su valor original seleccionando materiales de bajo impacto (González y García Navarro 2006). Rodríguez Serrano et al. (Rodríguez Serrano y Porras Álvarez, 2016) concluyen que el mayor impacto de las emisiones de la urbanización y los edificios tienen lugar durante la construcción y, por lo tanto, los ahorros posteriores debido a las reducciones de OE son muy modestos en comparación. Como Abanda et al. Sostienen (Abanda, Oti y Tah 2017) es crucial diseñar sistemas automatizados para calcular las emisiones de EE y $\mathrm{CO}_{2}$ en los edificios de acuerdo con las bases de datos estándar de medición y fijación de precios consolidados.

Mientras que la industria de la construcción genera el 35\% del total de residuos industriales en todo el mundo, la industria manufacturera de la UE consume el $40 \%$ de los recursos naturales (Mercader Moyano, M. 2010), pero solo se recupera el 25\% del CDW generado (AIE 2013). El Real Decreto Español 105/2008 (Gobierno español - Ministerio de la Presidencia, 2008) ya ha regulado la producción y gestión de CDW, teniendo en cuenta el Catálogo Europeo de Residuos (Diario Oficial de las Comunidades Europeas y
Decisión 2001/118/CE de residuos 2001). El II Plan Español de CDW para 2008/2015 mostró que menos del 18\% de la actividad de construcción e infraestructura CDW es reciclada (Ministerio de Medio Ambiente y Asuntos Rurales y Marinos 2008). Además, Solís considera que solo el $15 \%$ de CDW se recicla en España, lejos de los logros de otros miembros europeos (Solís Guzmán, J., 2010).

Los métodos para articular los datos de todo el proceso de construcción aún se están desarrollando, aunque la consideración de los problemas ambientales durante la etapa de diseño sigue siendo uno de los mayores desafíos para los diseñadores. Además, el Comité Europeo de Normalización recomienda integrar la producción del edificio desde una perspectiva ambiental para cumplir con las Directrices Europeas sobre Sostenibilidad en las Obras de Construcción (Comité Europeo de Normalización CEN 2012). La Directriz de la Unión Europea 2014/24/ UE sobre contratación pública establece que "para los contratos de obras públicas y concursos de diseño, los Estados miembros pueden exigir el uso de herramientas electrónicas específicas, como herramientas de modelado electrónico de información de construcción o similares". España ("España lanza la estrategia BIM con Mandato Penciled-in 2018 | BIM +" 2018), el Reino Unido (AEC (Reino Unido)), Alemania (ZukunftBAU 2013) y Francia (B. Delcambre) ya han comenzado a transponer estas Directrices a sus regulaciones de gobiernos locales. Pero aún quedan barreras importantes cuando se hace referencia a la industria AEC: accesibilidad a datos ambientales, conocimiento experto demasiado alto y difícil identificación de componentes o materiales alternativos apropiados (Bey, Hauschild y McAloone 2013).

El Building Information Modeling (BIM) se usa cada vez más en el sector de AEC para ofrecer planificación previa integrada, diseño y gestión de proyectos principalmente para edificios nuevos (Mousa, Luo y McCabe 2016). Una revisión de herramientas basadas en BIM para la evaluación del impacto ambiental mostró que la mayoría de ellas necesitan combinar el software BIM con otras aplicaciones para obtener la cuantificación de los indicadores ambientales. Una revisión sobre la integración BIM-sostenibilidad realizada durante todo el ciclo de vida del edificio señaló que la sostenibilidad ambiental, social y económica considera la etapa de diseño como el núcleo del asunto (Chong, Lee y Wang 2017). Otros autores revisaron la investigación sobre el análisis de integración BIM/LCA y sus posibilidades de simplificación en términos de datos de entrada-salida y resultados de LCA (Soust-Verdaguer, Llatas y García-Martínez, 2017) y señalaron que la mejor solución sería permanecer dentro del entorno BIM para facilitar la interacción entre el diseño y el desempeño ambiental (Antón y Díaz 2014). La mayoría de las herramientas revisadas consisten en aplicaciones para conectar el modelo BIM con datos ambientales como Tally ("Tally" 2018) y Revit (Najjar et al. 2017). Marzouk et al. propuso una combinación de varias herramientas de software: Autodesk Revit ("Familia Revit 


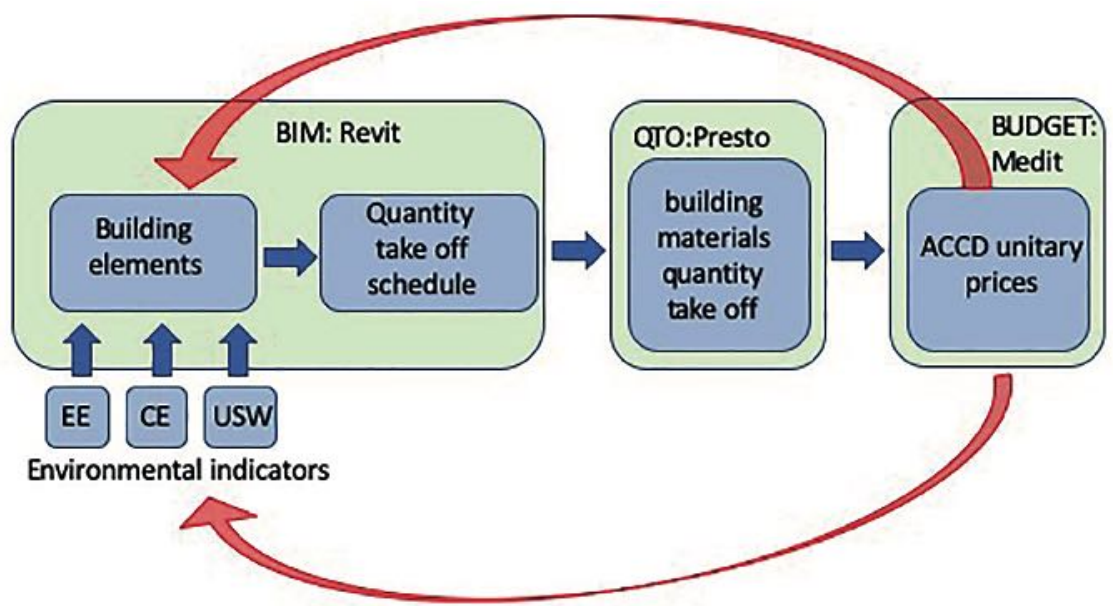

Figura 1. Indicadores ambientales integrados en familias y tipos BIM Fuente: los autores

I Software BIM | Autodesk", n.d.), link Revit DB ("Acerca de Autodesk Revit DB Link I Productos Revit I Autodesk Knowledge Network", n.d.), Microsoft Access ("Software y aplicaciones de bases de datos I Microsoft Access," n.d.) y ATHENA Impact Estimator ("IE for Buildings I Athena Sustainable Materials Institute," n.d.) al medir las emisiones directas e indirectas de carbono (CE, por su sigla en inglés) en proyectos de construcción (Marzouk, Abdelkader y AlGahtani 2017). Chen y Pan (Chen y Pan 2015) presentaron una toma de decisiones de criterios múltiples en medidas de construcción bajas en carbono, combinando Revit, eQuest ("EQUEST" 2018) y Promethee (Gul et al. 2018). Ajayi et al. (Ajayi et al. 2015) exploran una combinación de Revit Architecture, el complemento Green Building Studio (GBS) y ATHENA para el potencial de calentamiento global (GWP, por su sigla en inglés) y la evaluación del impacto en la salud. Azhar et al. (Azhar et al. 2011) combinaron Revit con IES Virtual Environment ("Presentación del software IESVE | Soluciones ambientales integradas" 2018) para calcular las emisiones de carbono y la energía incorporada para convertirlas en créditos LEED ("LEED I USGBC" 2018). Basbagill et al. (Basbagill et al. 2013) desarrollaron un modelo BIM utilizando DProfiler (Chelsea 2018) vinculado con eQuest ("eQUEST", n.d.) dentro de un entorno BIM mientras los resultados se cargan manualmente en SimaPro y Athena EcoCalculator para obtener CE. Ilhan et al. (Ilhan y Yaman 2016) desarrollaron una herramienta de evaluación de edificios ecológicos, utilizando Graphisoft ArchiCAD® vinculado a la base de datos de materiales BREEAM. Inyim et al. (Inyim, Rivera y Zhu 2015) presentaron Simulación de Impacto Ambiental de la Construcción (SimulEICon), que es una extensión BIM diseñada para ayudar en la etapa de diseño del proceso de toma de decisiones de un proyecto de construcción.

Gan et al. (Gan et al. 2018) desarrollaron un enfoque holístico para evaluar el carbono incorporado y operativo en edificios de gran altura, utilizando Revit y un complemento paramétrico, Dynamo. En una investigación posterior, el mismo equipo evaluó la reducción de CE al reemplazar el acero y el cemento por materiales reciclados (Gan et al. 2017). Yang et al. (Yang et al. 2018) desplegaron un flujo de trabajo unidireccional para calcular la operación y la energía de los materiales y $\mathrm{CO}_{2}$ ec.

Como Wong et al. señaló las herramientas BIM deben incluir el concepto de tres $\mathrm{R}$ (reducir, reutilizar y reciclar) en el análisis de impacto ambiental para proyectos nuevos y de modernización (Wong y Zhou 2015) para lograr objetivos de sostenibilidad. Además, CDW y USW deben tenerse en cuenta en una visión general de sostenibilidad porque afectan el equilibrio EE/CE durante el proceso de construcción. Cheng y Ma (Cheng y Ma 2013) desarrollaron un administrador de aplicaciones adicionales para CDW. Yehesis et al. (Yeheyis et al. 2013) propusieron un marco conceptual de gestión de CDW para maximizar las $3 R$ y minimizar la eliminación de residuos de construcción por LCA de proyectos de construcción BIM. Sáez et al. (Villoria Sáez et al.2018) señalaron que la mayor cantidad de CDW corresponde a la envoltura vertical en la modernización del edificio.

También se revisó la integración de los modelos BIM con métodos de medición estándar para obtener la cuantificación de EEy CE. Abanda etal. (Abanda, Otiy Tah 2017) desarrollaron una aplicación específica para vincular los modelos BIM con las Nuevas Reglas de Medición (NMR, por su sigla en ingles), el estándar del Reino Unido para la contratación pública (Surveyors 2018), utilizando Bath Inventory of Carbon and Energy (Bath ICE) ( Jones, C. y Allen, Stephen 2017).

Lützkendorf et al. expresó que la interoperabilidad con otro software para lograr la cantidad de material y la estimación de costos acelerarán el proceso de diseño, permitirán la comparación de soluciones alternativas y producirán mejores resultados (Lützkendorf et al. 2015).

Sin embargo, el software BIM aún debe cumplir con los requisitos de los diseñadores de edificios (Lamé, Leroy y Yannou 2017). Una de las principales barreras que enfrentan 


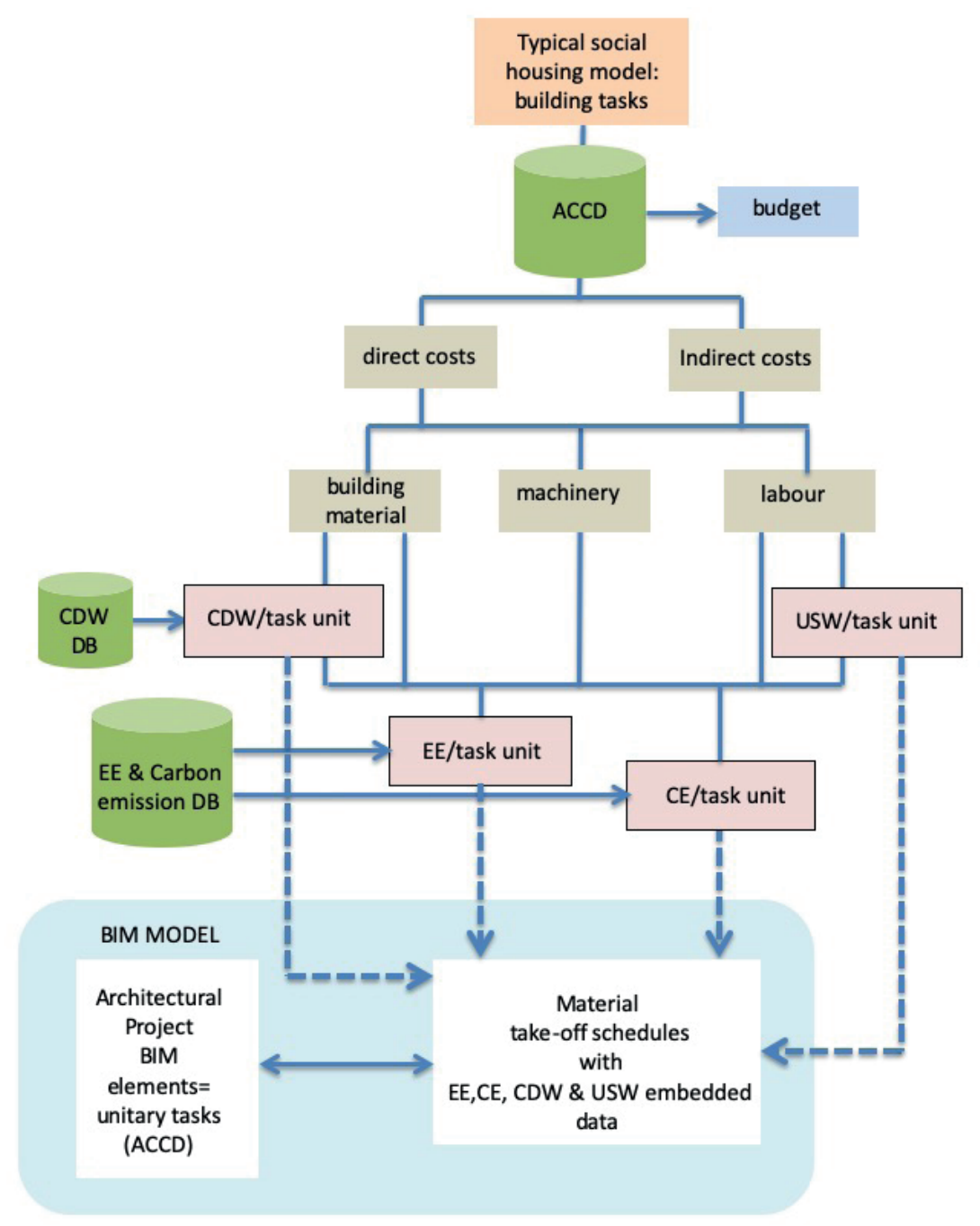

Figura 2: Flujo de trabajo metodológico sintetizado. Fuente: los autores

los diseñadores al entregar proyectos de construcción ecoeficientes es la experiencia específica para lidiar con diferentes software, bases de datos y metodologías. A pesar de esto, existe una tendencia creciente a integrar sistemas de evaluación, bases de datos, consultores y proveedores (Fiès, Lützkendorf y Balouktsi 2013). Mientras que algunos diseñadores están considerando la aplicación de herramientas complejas de datos y evaluación, otros sienten que todavía están sobrecargados.

El objetivo de este estudio es llenar el vacío de la evaluación del impacto ambiental en el diseño conceptual a través de indicadores BIM integrados. Esta estrategia facilita la elección del diseñador sobre las mejores alternativas para reducir el impacto ambiental en un marco abierto. El trabajo propone un enfoque ascendente para la cuantificación de CDW, USW, EE y CE de acuerdo con la estructura de desglose del trabajo (WBS, por su sigla en inglés) de la ACCD (por su sigla en inglés) (Fig.1). Presto ${ }^{\circ}$ y Medit $₫$ utilizan los elementos de los edificios BIM para extraer la lista de cantidades y costos ("Acae Presto" 2018) ("Medit Mediciones en BIM con REVIT" 2013).

\section{MATERIALES Y MÉTODOS}

La metodología es apropiada para el diseño conceptual aunque la definición del material no esté completamente definida porque la ACCD proporciona un catálogo completo de sistemas y materiales de construcción que están disponibles como elementos de Revit.El flujo de trabajo se divide en tres pasos para cuantificar CDW, USW, EE y CE de un edificio nuevo o modernizado (Fig. 2):

Descomposición de cada capítulo de la base de datos de costos de construcción en sus niveles inferiores: materiales de construcción, mano de obra y maquinaria, que incluye las tareas auxiliares y los costos indirectos.Adición de parámetros ambientales: CDW, EE, CE y USW a la WBS de ACCD para obtener los indicadores.

Incorporación de indicadores ambientales en los programas de despegue de materiales BIM para cuantificar EE, CE, CDW y USW. 


\section{DESCOMPOSICIÓN DE TAREAS DE CONSTRUCCIÓN}

La normativa española actual se compromete a entregar nuevos proyectos de construcción en formato de archivo BIM, utilizando CCD para licitaciones públicas. Andalucía cuenta con la $A C C D$, que es una base de datos en línea descargable gratuita. Proporciona una descripción detallada y una medición de todas las tareas realizadas a lo largo del proceso de construcción, mientras que los archivos pueden combinarse con QTO y formatos de software de cálculo de costos (Asociación FIE-BDC).

El ACCD divide las tareas de construcción en capítulos que cuantifican el material de construcción, la mano de obra y la maquinaria llamados COSTOS DIRECTOS (DC, por su sigla en inglés) al tiempo que agrega un porcentaje para las tareas generales llamadas COSTOS INDIRECTOS (IC, por su sigla en inglés) (Barón, J. et al.2017) que afectan el conjunto del proceso de construcción como iluminación general, electricidad y combustible para maquinaria y trabajo de personal técnico y auxiliar. Las tareas auxiliares se desglosan de las tareas principales.CDW se desarrolla en capítulos específicos: uno para cada tipo de desecho (mezclado, madera, papel y cartón, acero, suelo inerte, entre otros). Cada capítulo comprende los elementos que componen una tarea medida en unidades específicas. Para la cuantificación de CDW, aplicamos la metodología desarrollada por Ramírez de Arellano (Ramírez de Arellano Agudo 2002), incluida en el ACCD en 2017 y basada en el Decreto Nacional para regular la producción y gestión de CDW (Gobierno español - Ministerio de la Presidencia, 2008) y en la Lista Europea de Residuos 2000 (Diario Oficial de las Comunidades Europeas y Decisión 2001/118/CE de residuos de la Comisión 2001). La metodología se describe completamente en (MercaderMoyano y Ramírez-de-Arellano-Agudo 2013) y (Marrero y Ramirez de Arellano, 2010).

\section{ADICIÓN DE LOS PARAMETROS AMBIENTALES: EE, CE, USW Y CDW A ACCD WBS}

El procedimiento utiliza el presupuesto de ACCD como marco para integrar los indicadores ambientales (Freire Guerrero, A. y Marrero Meléndez, M. 2015). Los datos se dividen en dos ramas principales: costos directos (materiales de construcción, maquinaria, mano de obra) y costos indirectos (electricidad general, agua y desechos).

Los valores EE y CE se extrajeron de la base de datos Ecoinvent (Centro Suizo para Ciclo de Vida e Inventarios 2017), BEDEC (Instituto de Tecnología de la Construcción de Cataluña 2017). Los valores de EE y USW de alimentos para el personal se extrajeron de la investigación (Solís Guzmán, J., 2010) y (Freire Guerrero, Antonio 2017). Las emisiones de $\mathrm{CO}_{2}$ de la electricidad se obtuvieron de la matriz energética andaluza cuya mezcla de electricidad produjo $419,9 \mathrm{tCO}_{2} / \mathrm{GWh}$, equivalente a $0.117 \mathrm{kgCO}_{2} / \mathrm{MJ}$ en 2016 (Agencia Andaluza de la Energía 2017).

\section{Adición de parámetros ambientales a los costos directos}

A continuación se muestran las ecuaciones de costos directos (DC) de cada precio unitario. La Ecuación 1 muestra la EE de cada artículo que integra un precio unitario.

$$
E E_{D C}=\sum_{i \in I} E E_{i}
$$

I=\{material de construcción, maquinaria, alimentos para el personal, USW, CDW por tipo\}

$\mathrm{EE}_{=}$tarea básica $\mathrm{EE}$ en $\mathrm{MJ}$

Como los materiales de construcción se pueden medir en $\mathrm{m}^{3}, \mathrm{~kg}$, ton $\circ \mathrm{m}^{2}$, entre otros, la EE debe convertirse a la unidad objetivo correspondiente (MJ/TU) (Tabla 1).

\begin{tabular}{|c|c|c|c|}
\hline $\begin{array}{c}\text { Material de } \\
\text { construcción }\end{array}$ & unidad & EE MJ/TU & $\begin{array}{c}\text { CE kgCO2/ } \\
\text { TU }\end{array}$ \\
\hline ACETO & $\mathrm{kg}$ & 40.00 & 2.80 \\
\hline $\begin{array}{c}\text { HA-25/B/20/lla } \\
\text { HORMIGÓN }\end{array}$ & $\mathrm{m} 3$ & 6209.97 & 159.46 \\
\hline MADERA DE PINO & $\mathrm{m} 3$ & 3.00 & 714.00 \\
\hline $\begin{array}{c}\text { LADRILLO HUECO } \\
\text { CERÁMICO }\end{array}$ & millas & 5290 & 487.60 \\
\hline ARENA & $\mathrm{m} 3$ & 247.51 & 48.00 \\
\hline
\end{tabular}

Tabla 1. EE y CE según unidades de precio unitario ACCD Fuente: los autores

La maquinaria se calcula en horas dependiendo de la $E E / h$ de cada tipo de máquina (Ec. 2).

$$
E E_{m}=\sum_{m \in M} Q_{m} x E E_{m} / h
$$

$M=\{c a m i o ́ n$ basculante, cargador frontal, retroexcavadora, vibrador, grúa horquilla\}

La cuantificación de USW se calcula con un valor fijo por hora laboral $(0.077 \mathrm{~kg} / \mathrm{h})$

La mano de obra se calcula en horas para calcular $\mathrm{EE}_{\text {worker }}$ food $\mathrm{EE}_{\text {usw }}$ (Eq. 3-4)

(3) $E E_{\text {staff food }}=\sum_{i=1}^{n} Q_{i} x E E_{\text {worker food }} / h$

$$
E E_{U S W}=\sum_{i=1}^{n} Q_{i} x E E_{U S W} / h
$$

Q = duración de la ejecución de una tarea básica en h para el trabajador $\mathrm{i}$

$\mathrm{n}=$ trabajadores

$\mathrm{EE}_{\text {worker food }} / \mathrm{h}=\mathrm{EE}$ por hora de trabajo

$\mathrm{EE}_{\mathrm{Usw}} \mathrm{wh}=$ USW por hora de trabajo 
CDW se calcula en diferentes unidades de acuerdo con la eliminación de residuos (Ec. 5).

$$
W_{o \in O}=C_{R o} x C_{C o} x C_{T o}
$$

$W_{\text {o }}$ es el coeficiente resultante, uno por cada tipo de desechoO=\{cartón/papel, madera, suelo inerte, aluminio, cobre, bronce, hierro, acero, agregado árido/piedra, plástico/sintético, vidrio y residuos mezclados\}

La cantidad efectiva de residuos se muestra en Ec. 6.

$$
E E_{C D W}=\sum_{b \in B} Q_{b} x W_{o} x R F_{o} x E E_{o}
$$

$\mathrm{Q}_{b}$ es la cantidad de material de construcción

$\mathrm{B}=\{$ materiales de construcción $\}$

$\mathrm{RFo}=$ factor de reciclaje que reduce la $\mathrm{EE}$

$\mathrm{EE}_{\mathrm{o}}=\mathrm{EE}$ unitaria por tipo de desecho

La emisión de carbono de DC (CE $\left.{ }_{D C}\right)$ se muestra en la Ec. 7.

$$
C E_{D C}=\sum_{i \in I} C E_{i}
$$

I=\{material de construcción, maquinaria, alimentos para el personal, USW, CDW por tipo\}

$\mathrm{CE}_{\mathrm{i}}$ es la tarea básica $\mathrm{CE}$ en $\mathrm{kgCO}_{2}$

CE/alimento $\mathrm{kg}$ se fija como $0.24 \mathrm{kgCO} /$ /alimento $\mathrm{kg}$ (Ec. 8 y Ec. 9).

$$
\begin{aligned}
C E_{\text {staff food }} & =\sum_{i=1}^{n} Q_{i} x C E_{\text {worker food }} / h \\
C E_{U S W} & =\sum_{i=1}^{n} Q_{i} x C E_{U S W} / h
\end{aligned}
$$

$\mathrm{Q}_{\mathrm{i}}=$ ejecución de tareas básicas en $\mathrm{h}$ para trabajador $\mathrm{i}$ $\mathrm{n}=$ trabajadoresCE $\mathrm{E}_{\text {worker food }} / \mathrm{h}=\mathrm{CE}$ generado por hora laboralCE $\mathrm{USW}_{\mathrm{W}} / \mathrm{h}=\mathrm{CE}$ USW generado por hora laboral En el caso de CDW, agregamos un factor de reciclaje RF (por su sigla en inglés), que depende del tipo de desecho y reduce la CE (Ec. 10):

$$
C E_{C D W}=\sum_{b \in B} Q_{b} x W_{o} \times R F_{o} \times C E_{o}
$$

$\mathrm{RF}_{\mathrm{o}}=$ factor de reciclajeCE $\mathrm{E}_{\mathrm{o}}=$ emisiones de carbono unitarias por tipo de residuo

\section{Adicion de parametros ambientales a los costos indirectos}

Los costos indirectos (IC) son un porcentaje fijo de los costos directos: el 10,62\% de DC se adopta para la vivienda social típica en Andalucía.

El consumo de energía se divide en tres categorías: personal general, electricidad y combustible, de acuerdo con el análisis típico de IC de vivienda social. (Ec. 11).

\section{(11) $E E_{I C}=\sum_{s \in S} E E_{I C s} x I C_{s}+\sum E E_{I C S U W} / T U$}

$\mathrm{EE}_{\mathrm{ICs}}=\mathrm{IC} \mathrm{EE}$ in $\mathrm{MJ}$

$\mathrm{IC}_{\mathrm{S}}=$ porcentaje correspondiente $S=\{$ personal, electricidad, combustible\}

$E E_{I C ~ U S W}=I C$ EE del consumo de alimentos del personal USW en MJ

El EE de $C_{\text {IC }}$ se calcula de la misma manera que una tarea unitaria de IC.

\section{ACCD PRECIO UNITARIO + INDICADORES AMBIENTALES}

El nueva WBS de PRECIO UNITARIO ACCD + INDICADORES AMBIENTALES (EUP) comprende la adición de EE, CE, USW y CDW (Fig. 3)

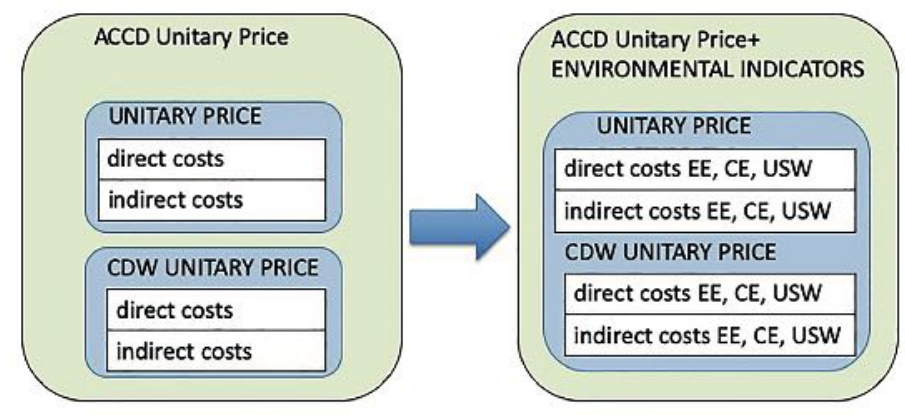

Fig. 3. WBS de precios unitarios de ACCD y precios unitarios ambientales (EUP), que muestra la adición de los indicadores ambientales y la fusión del precio unitario principal con los precios unitarios CDW correspondientes Fuente: los autores

La relación entre EE, CE, CDW y USW y EUP calcula los indicadores EE, CE, CDW y USW (Ec. 12-15).

$$
\begin{gathered}
E E_{E U P} \text { indicator }(M J / E U P u n i t)=\frac{E E_{E U P}+E E_{C D W E U P}}{E U P U} \\
C E_{E U P} \text { indicator }\left(\mathrm{kgCO}_{2} / \text { EUPunit }\right)=\frac{C E_{E U P}+C E_{C D W E U P}}{E U P U} \\
C D W_{E U P} \text { indicator }\left(C D W u n i t / E U P U \text { it }=\frac{C D W_{C D W E U T}}{E U P U}\right. \\
U S W_{E U P} \text { indicator }=\frac{U S W_{E U P}+U S W_{C D W E U P}}{E U P U}
\end{gathered}
$$

EUP = unidad de precio unitario ambiental

CDW EUPu $=$ CDW unidad de precio unitario ambiental

\section{COEFICIENTES AMBIENTALES QUE SE INCORPORAN EN EL MODELO Y CUANTIFICACIÓN BIM}

Luego, los indicadores ambientales se integran como nuevos parámetros en cada familia BIM. Los elementos BIM se miden en las mismas unidades que los EUP. Autodesk 
Revit ${ }^{1}$ genera los horarios de descuento de materiales para cada tipo de elemento, por lo que podemos agregar parámetros ambientales: valores EE, CE, CDW y USW por unidad. Finalmente, el programa calcula las cantidades totales para cada parámetro.

\section{ESTUDIO DE CASO}

\section{UN BLOQUE DE VIVIENDA SOCIAL: LA ESTRUCTURA DE HORMIGÓN REFORZADA}

Esta investigación desarrolla la cuantificación de los indicadores ambientales aplicados a la estructura de hormigón armado de un bloque de viviendas sociales típico en Sevilla (Fig. 4) en el diseño conceptual. Esta tipología representa una de las más difundidas en Andalucía y se ha descrito ampliamente (Mercader Moyano, M. 2010; González-Vallejo, Marrero y SolísGuzmán 2015). Este tipo de edificios generalmente tiene una estructura de hormigón reforzado de 2/4 pisos, carpintería de aluminio y techo de losa plana (Marrero Meléndez, M. et al. 2015). La estructura de hormigón armado representa un gran impacto ambiental (De Wolf, Pomponi y Moncaster 2017).

\section{INDICADORES AMBIENTALES}

Como ejemplo, la Tabla 2 muestra la adición de indicadores ambientales a cada precio unitario para la capa de hormigón de limpieza.

\section{MODELO BIM}

El plan de piso es un cuadrado lateral de $23 \mathrm{~m}$ y el edificio tiene dos pisos que suman $1.058 \mathrm{~m}^{2}$. Vinculamos una tarea unitaria ACCD a cada elemento de construcción. Por lo tanto, ciertos elementos de construcción, que no tienen ningún elemento BIM equivalente como encofrados o excavaciones, se dibujan como elementos nuevos: superficies para medir áreas de encofrado para elementos estructurales (pilares, escaleras, losas y vigas) y cajas para excavaciones (Fig. 5 ).

\section{INCORPORACIÓN DE INDICADORES AMBIENTALES}

La Tabla 3 sintetiza los coeficientes ambientales por tarea unitaria de la estructura del edificio integrados en el modelo BIM como nuevos parámetros.

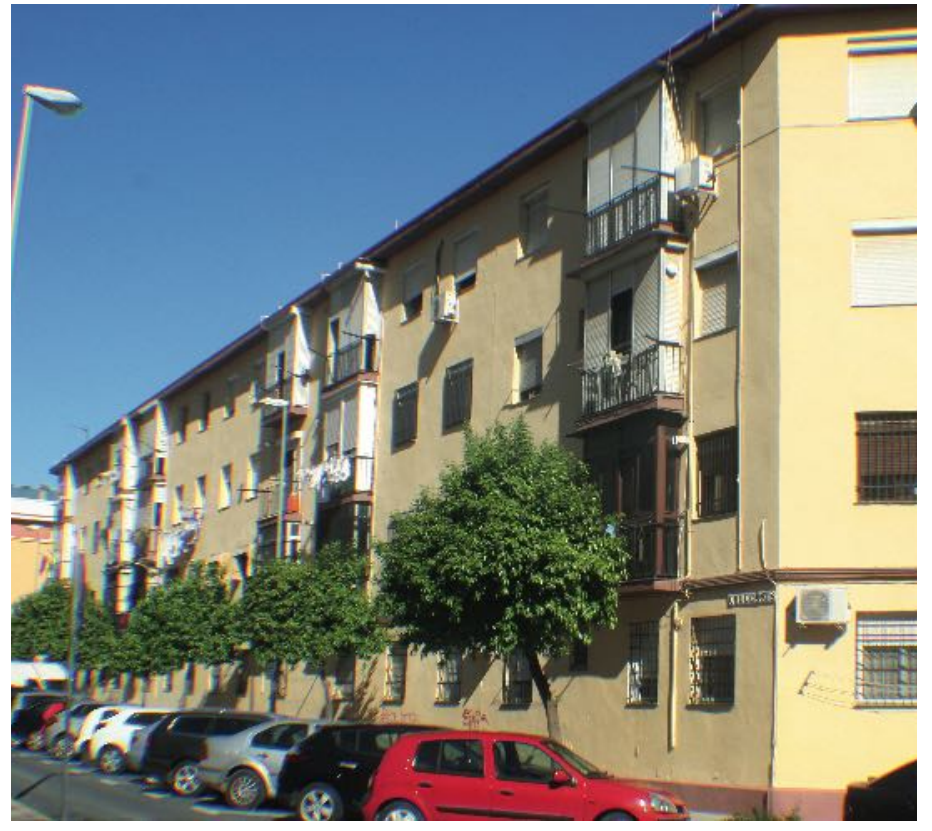

Fig. 4: Bloque de viviendas sociales en Sevilla Fuente: los autores

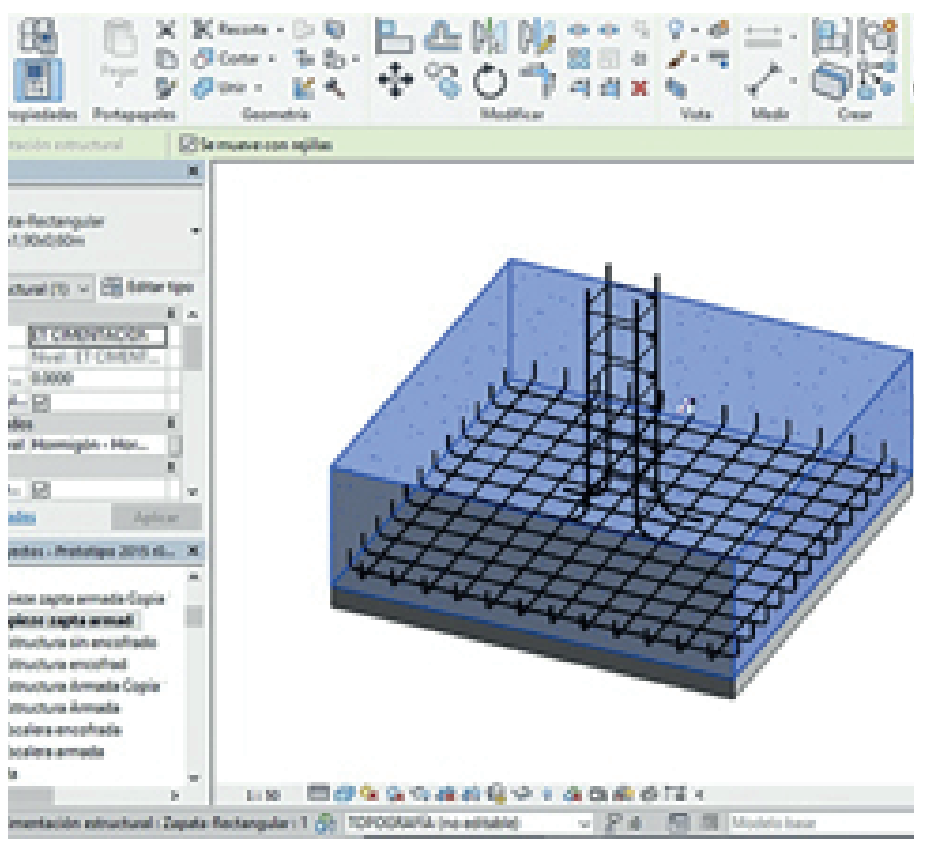

Fig. 5. Captura de pantalla de Revit que muestra los elementos BIM en correspondencia con el precio unitario ACCD de losa de cimentación y tapa de pilote Fuente: los autores 


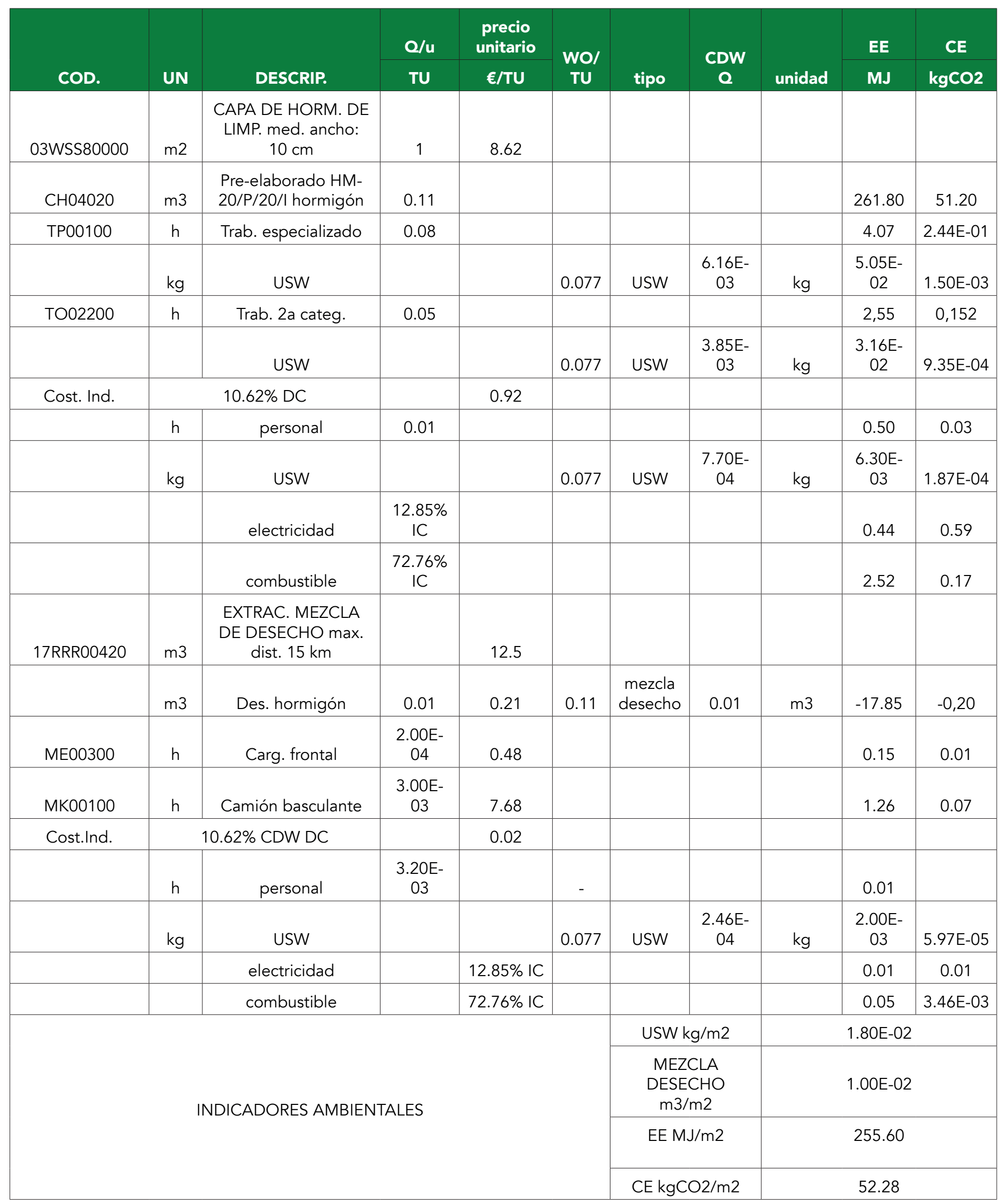

Tabla 2. Capa de hormigón de limpieza. Med. ancho: $10 \mathrm{~cm}$ Fuente: modificado de ACCD por los autores 


\begin{tabular}{|c|c|c|c|c|c|c|c|c|c|c|}
\hline \multirow[b]{2}{*}{ TASK GROUP } & \multirow[b]{2}{*}{ UNITARY TASK } & \multirow[b]{2}{*}{ unit } & \multicolumn{6}{|c|}{ CDW coefficients } & \multirow[b]{2}{*}{$\begin{array}{c}\mathrm{EE} \\
\mathrm{MJ} / \mathrm{u}\end{array}$} & \multirow[b]{2}{*}{$\begin{array}{c}\mathrm{CE} \\
\mathrm{kgCO} 2 / \mathrm{u}\end{array}$} \\
\hline & & & $\begin{array}{l}\text { USW } \\
\mathrm{kg} / \mathrm{u}\end{array}$ & $\begin{array}{l}\text { wood } \\
\text { ton/u }\end{array}$ & $\begin{array}{l}\text { inert } \\
\text { soil } \\
\text { ton } / \mathrm{u}\end{array}$ & $\begin{array}{l}\text { paper } 8 \\
\text { cardboard } \\
\mathrm{kg} / \mathrm{u}\end{array}$ & $\begin{array}{l}\text { steel } \\
\text { ton/u }\end{array}$ & $\begin{array}{l}\text { mixed } \\
\mathrm{m} 3 / \mathrm{u}\end{array}$ & & \\
\hline $\begin{array}{l}\text { DEMOLITION } \\
\text { \& PREVIOUS } \\
\text { WORKS }\end{array}$ & $\begin{array}{c}\text { MECHANICAL } \\
\text { LAND CLEARING }\end{array}$ & $\mathrm{m} 2$ & $\begin{array}{c}4.64 \mathrm{E}- \\
04\end{array}$ & 8.00E-02 & & & & & 19.92 & 1.26 \\
\hline \multirow{3}{*}{$\begin{array}{c}\text { LAND } \\
\text { CODITIONING }\end{array}$} & $\begin{array}{c}\text { BOX } \\
\text { EXCAVATION, } \\
\text { MEDIUM } \\
\text { CONSISTENCY } \\
\text { SOIL }\end{array}$ & m3 & $\begin{array}{c}3.36 \mathrm{E}- \\
03\end{array}$ & & 1.25 & & & & 211.60 & 13.03 \\
\hline & $\begin{array}{l}\text { FOUNDATION } \\
\text { SLAB } \\
\text { MECHANICAL } \\
\text { EXCAVATION, } \\
\text { MED. CONSIT., } \\
\text { max. depth: } 4 \mathrm{~m}\end{array}$ & m3 & $\begin{array}{l}1.31 \mathrm{E}- \\
02\end{array}$ & & 1.25 & & & & 267.97 & 17.57 \\
\hline & $\begin{array}{c}\text { TRENCH } \\
\text { MECHANICAL } \\
\text { EXCAVATION, } \\
\text { max. depth: } \\
\text { 1.5m, width: } 40 \\
\text { cm }\end{array}$ & m3 & $\begin{array}{c}1.34 \mathrm{E}- \\
02\end{array}$ & & 1.25 & & & & 267,26 & 17.42 \\
\hline \multirow{5}{*}{ FOUNDATIONS } & $\begin{array}{c}\text { FOUNDATION } \\
\text { CORRUGATED } \\
\text { STEEL RODS } \\
\text { B500S }\end{array}$ & $\mathrm{kg}$ & $\begin{array}{c}1.70 \mathrm{E}- \\
03\end{array}$ & & & & 9.79E-06 & & 47.10 & 3.38 \\
\hline & $\begin{array}{c}\text { LOST BRICK } \\
\text { FORMWORK OF } \\
\text { RING BEAMS, } \\
\text { FOUNDATION } \\
\text { SLABS \& PILE } \\
\text { CAPS }\end{array}$ & $\mathrm{m} 2$ & $\begin{array}{c}1.88 \mathrm{E}- \\
02\end{array}$ & 2.60E-03 & & 20.60 & $2.60 \mathrm{E}-03$ & $\begin{array}{c}9.10 \mathrm{E}- \\
03\end{array}$ & 708.15 & 36.83 \\
\hline & $\begin{array}{c}\text { FOUNDATION } \\
\text { \& RING BEAM } \\
\text { CONCRETE HA- } \\
\text { 25/B/20/lla }\end{array}$ & m3 & $\begin{array}{l}4.26 \mathrm{E}- \\
02\end{array}$ & & & & & $\begin{array}{l}9.10 \mathrm{E}- \\
03\end{array}$ & 2326.53 & 531.32 \\
\hline & $\begin{array}{c}\text { FOUNDATION } \\
\text { SLAB \& PILE CAP } \\
\text { CONCRETE HA- } \\
\text { 25/B/20/lla }\end{array}$ & $\mathrm{m} 3$ & $\begin{array}{c}4.00 \mathrm{E}- \\
02\end{array}$ & & & & & 0.11 & 2516.52 & 304.78 \\
\hline & $\begin{array}{l}\text { CLEANING } \\
\text { CONCRETE } \\
\text { LAYER med. } \\
\text { width: } 10 \mathrm{~cm}\end{array}$ & $\mathrm{~m} 3$ & $\begin{array}{c}1,80 \mathrm{E}- \\
02\end{array}$ & & & & & 0,01 & 255,60 & 52.28 \\
\hline \multirow[b]{2}{*}{ STRUCTURE } & $\begin{array}{c}\text { CORRUGATED } \\
\text { STEEL RODS } \\
\text { B500S }\end{array}$ & $\mathrm{kg}$ & $\begin{array}{c}4.26 \mathrm{E}- \\
02\end{array}$ & & & & $1.08 \mathrm{E}-05$ & & 47.10 & 3.38 \\
\hline & $\begin{array}{c}\text { WOOD } \\
\text { FORMWORK } \\
\text { REMOVAL FROM } \\
\text { CONCRETE } \\
\text { ELEMENTS }\end{array}$ & $\mathrm{m} 2$ & $\begin{array}{c}1.54 \mathrm{E}- \\
02\end{array}$ & & & & & & 10.69 & 0.92 \\
\hline
\end{tabular}




\begin{tabular}{|c|c|c|c|c|c|c|c|c|c|}
\hline \multirow{7}{*}{ STRUCTURE } & $\begin{array}{c}\text { METAL PANEL } \\
\text { FORMWORK } \\
\text { REMOVAL FROM } \\
\text { CONCRETE } \\
\text { ELEMENTS }\end{array}$ & $\mathrm{m} 2$ & $\begin{array}{c}8.47 \mathrm{E}- \\
03\end{array}$ & & & & & 5.92 & 0.50 \\
\hline & $\begin{array}{l}\text { PINEWOOD } \\
\text { FORMWORK } \\
\text { FOR CONCRETE } \\
\text { SLABS }\end{array}$ & $\mathrm{m} 2$ & $\begin{array}{c}6.39 \mathrm{E}- \\
02\end{array}$ & 1.00E-06 & & $1,75 \mathrm{E}-04$ & & 80.55 & 10.57 \\
\hline & $\begin{array}{c}\text { METAL } \\
\text { FORMWORK } \\
\text { FOR PILLARS }\end{array}$ & $\mathrm{m} 2$ & $\begin{array}{c}2.54 \mathrm{E}- \\
02\end{array}$ & & & $2,00 \mathrm{E}-05$ & & 46.97 & 2.94 \\
\hline & $\begin{array}{c}\text { REINFORCED } \\
\text { CONCRETE HA- } \\
\text { 25/P/20/lia FOR } \\
\text { SLABS }\end{array}$ & m3 & $\begin{array}{c}6.80 \mathrm{E}- \\
02\end{array}$ & & & & 0,11 & 2344.39 & 486.19 \\
\hline & $\begin{array}{c}\text { REINFORCED } \\
\text { CONCRETE HA- } \\
\text { 25/P/20/lia FOR } \\
\text { PILLARS }\end{array}$ & m3 & $\begin{array}{l}5.10 \mathrm{E}- \\
02\end{array}$ & & & & 0,11 & 2044.20 & 328.82 \\
\hline & $\begin{array}{l}\text { WOOD } \\
\text { FORMWORK } \\
\text { REMOVAL }\end{array}$ & $\mathrm{m} 2$ & $\begin{array}{l}1.55 \mathrm{E}- \\
02\end{array}$ & & & & & 2.71 & 0.27 \\
\hline & $\begin{array}{l}\text { HOLLOW BRICK } \\
\text { WALL width: } 5 \mathrm{~cm}\end{array}$ & $\mathrm{~m} 2$ & $\begin{array}{c}4.39 \mathrm{E}- \\
02\end{array}$ & 3.40E-02 & 0.27 & 3.50E-06 & $\begin{array}{l}4.00 \mathrm{E}- \\
03\end{array}$ & 1014.83 & 85.13 \\
\hline
\end{tabular}

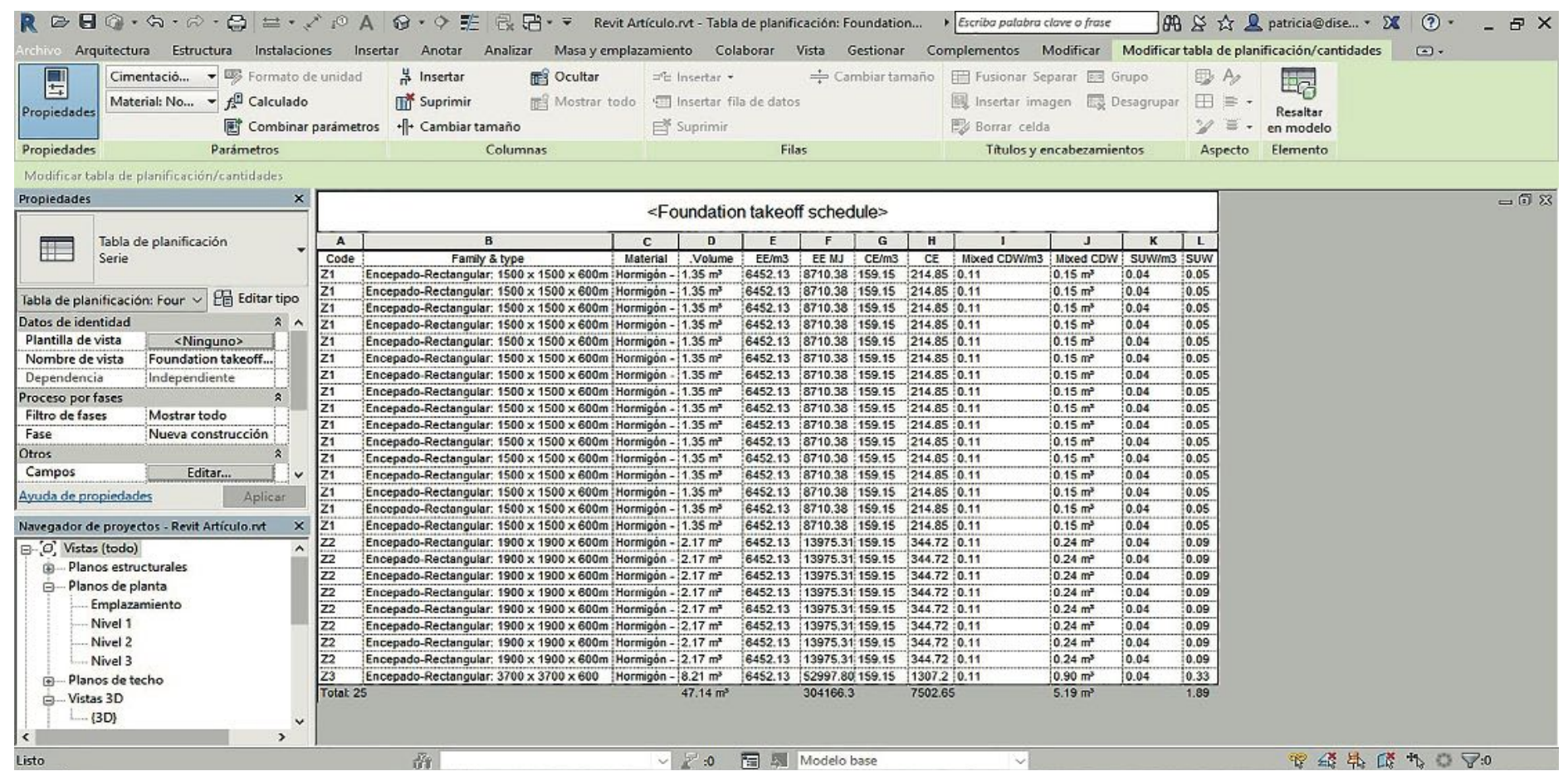

Fig. 6: Captura de pantalla de Revit que muestra el cronograma de despegue de material de la familia de losas de cimentación con datos ambientales integrados Fuente: los autores 

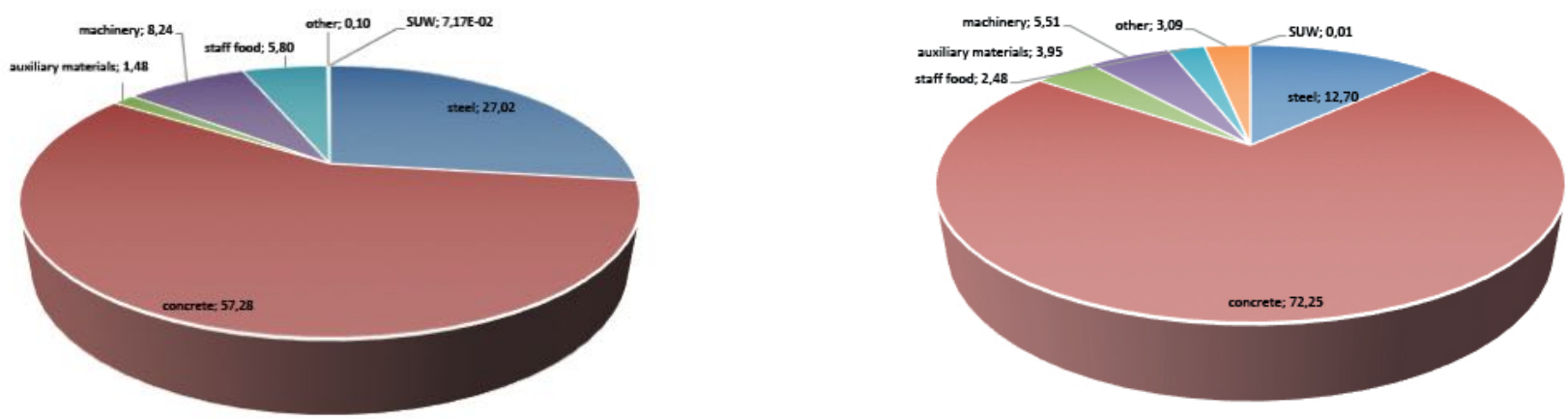

Fig. 7. Estructura de hormigón armado porcentajes EE Fuente: los autores Fig. 8. Estructura de hormigón armado Porcentajes CE Fuente: los autores

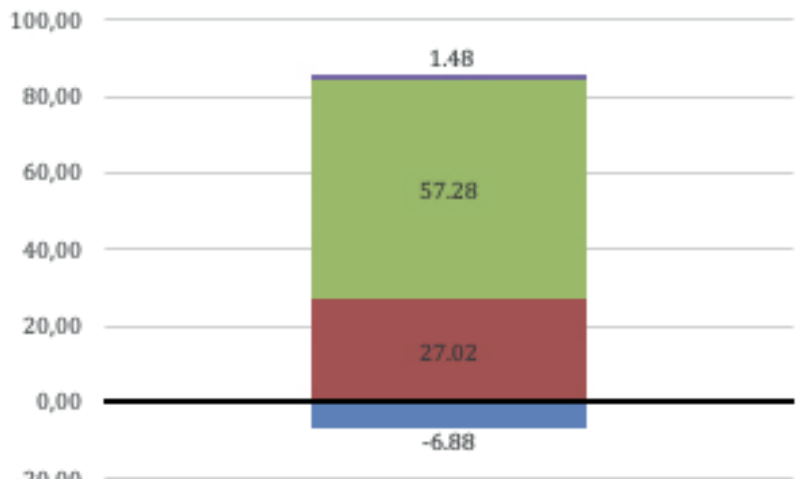

$-20,00$

$$
\text { Concrete } \quad \text { Steel CDW }
$$

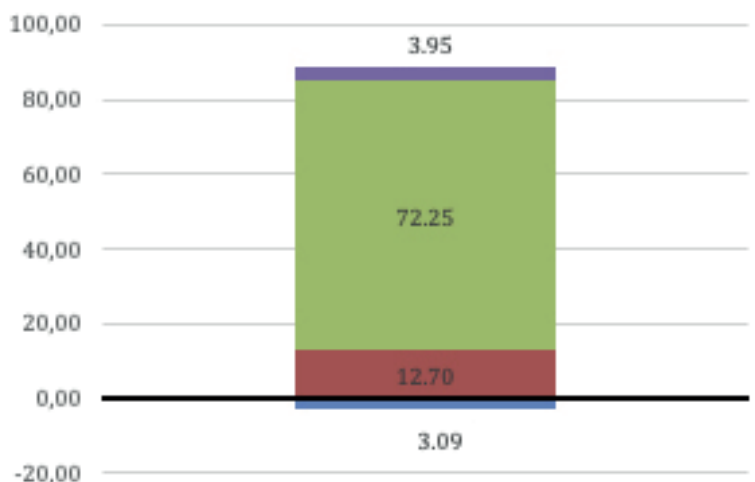

Fig. 9. Porcentajes de materiales de construcción EE y CDW Fuente: los autores Fig. 10. Porcentajes de materiales de construcción CE y CDW Fuente: los autores
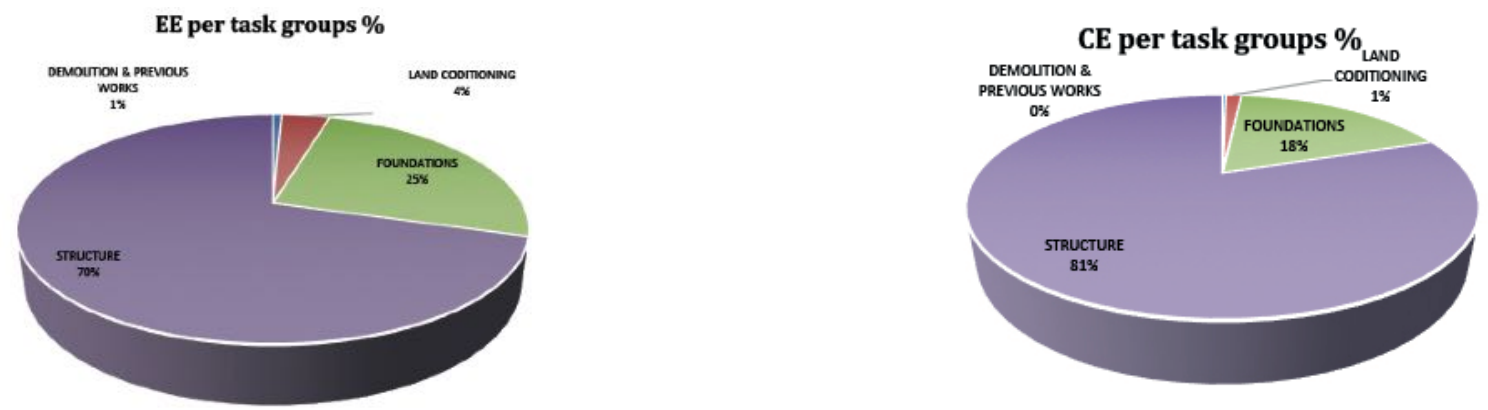

Fig. 11. Porcentajes de EE correspondientes a la estructura de soporte de carga de hormigón armado divididos por grupo de tareas Fuente: los autores Fig. 12: Porcentajes CE de estructura de hormigón armado divididos por grupo de trabajo Fuente: los autores

Después de dibujar todo el modelo, Revit extrae los Programas de descuento de materiales, enumerando los subcomponentes o materiales de cualquier familia de Revit. Los gráficos cuantifican USW, CDW, EE y CE, para cada familia de Revit en tiempo real, reflejando cualquier cambio realizado en el modelo. La figura 6 muestra el calendario de despegue de losas de cimentación y tapas de pilotes.

\section{RESULTADOS Y DISCUSIÓN}

Los materiales de construcción, USW, CDW, EE y CE se cuantifican dentro de Revit. Los resultados se analizan después de extraer los horarios de descuento de material de cada elemento BIM, de acuerdo con EUP (Fig. 7-8). En las figuras 9 y 10, la reducción de EE-CE debido al reciclaje de CDW se calculó considerando que solo el 15\% de los residuos 
se recicla en España (Solís Guzmán, J. 2010). Como se esperaba, la reducción de CDW EE-CE no es considerable: $-6.88 \%$ y $3.09 \%$ respectivamente.

Al analizar los grupos de tareas, EE y CE muestran resultados casi similares (70\%) (Fig. 11-12). Estos grupos de tareas comprenden no solo el acondicionamiento del terreno, las excavaciones y la construcción, sino también las tareas de CDW. La división en grupos de tareas que se toma de $A C C D$, le permite al diseñador evaluar diferentes tipos de sistemas de construcción para cada grupo modificando el modelo BIM y cambiando los coeficientes posteriores en los horarios de despegue del material. Podemos inferir que la superestructura juega un papel clave para lograr una disminución sustancial de EE y CE (Ferreiro-Cabello et al.2016).

El siguiente paso es cuantificar la estructura de soporte de carga CDW-USW para un bloque típico de vivienda social (TSH) por metro cuadrado (Fig. 13). Si el suelo inerte y la madera se dejan aparte, ya que EE y CE son casi nulos y pueden ser reciclables a bajo costo, la estructura de soporte de carga genera $75.77 \mathrm{~kg}$ de CDW mixto, $0.34 \mathrm{~kg}$ de acero CDW, $0.69 \mathrm{~kg}$ de papel y cartón CDW y $0.13 \mathrm{~kg}$ de USW por metro cuadrado.

\section{CONCLUSIONESYTRABAJOFUTURO}

El marco desarrollado en este estudio permite incorporar indicadores ambientales en los modelos BIM para cuantificación EE, CE, USW y CDW, siguiendo ACBS WBS. Si se aplica en el diseño conceptual, ya que BIM regenera la geometría en tiempo real, el diseñador puede evaluar diferentes soluciones formales y técnicas cuando las decisiones impactan más en LCA. En las etapas avanzadas de diseño, realizar modificaciones sustanciales al proyecto de construcción exige más tiempo y dinero que en las primeras etapas.

Los principales hallazgos de esta investigación son:

- El desarrollo de indicadores, lo que constituye una piedra angular de evaluación cuantitativa.

- La metodología que considera las necesidades del diseñador desde una perspectiva holística que fomenta la inclusión de criterios ambientales desde las primeras etapas de diseño al simplificar el proceso de introducción de indicadores.

- El cálculo del desempeño ambiental junto con el diseño arquitectónico, al reflejar todo cambio en un flujo de trabajo continuo que retroalimenta el proceso en tiempo real.

En este estudio de caso sobre una estructura de hormigón reforzado en bloques de viviendas, estos resultados verifican los supuestos de otros autores: es mejor usar materiales con bajas EE que con altas EE debido a la baja tasa de reciclaje (González y García Navarro, 2006). Además, la

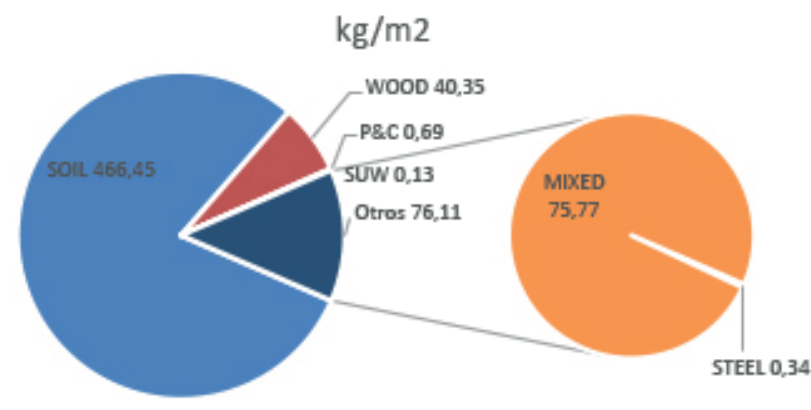

Fig. 13. Estructura de hormigón armado TSH CDW kg/m²- USW kg/m² Fuente: los autores

estructura de soporte de carga TSH genera $76.11 \mathrm{~kg} / \mathrm{m}^{2}$ de CDW; $75.77 \mathrm{~kg} / \mathrm{m}^{2}$ de CDW mixto, principalmente debido al hormigón y solo $0.34 \mathrm{~kg} / \mathrm{m}^{2}$ de acero CDW. Cuando se consideran por tarea, las fundaciones presentan un impacto mucho menor que la superestructura.

Estos resultados ofrecen una brecha para reducir el impacto ambiental al innovar las soluciones de estructura portante. Los materiales con baja EE/CE como la madera o los agregados inertes reciclados pueden ofrecer buenas alternativas. El sistema de construcción tradicional andaluz debe reformularse para reducir el agotamiento de los recursos naturales, el consumo de energía y la generación de CE y USW/CDW.

Una de las limitaciones de estos indicadores es que están adaptados a WBS de ACCD, ya que fueron creados para mejorar los modelos andaluces de Revit para obtener la lista de cantidades y costos a través de otros programas como Presto y Medit.En el trabajo futuro, se espera extender los indicadores ambientales a cada tarea unitaria para obtener una evaluación ambiental completa de la construcción del edificio que permita la comparación entre diferentes sistemas de construcción y materiales dentro del entorno BIM. Cuando se agreguen a ACCD, estarán disponibles para los usuarios sin una gran experiencia en herramientas de evaluación de impacto ambiental, lo que hace que esta tarea sea menos propensa a errores y consuma menos tiempo.

Una futura línea de investigación puede incluir la aplicación de la metodología a un conjunto de proyectos representativos para extender los resultados a nivel nacional y la adición de los resultados ambientales a los sistemas de certificación como BREAM y LEED. Otra línea de investigación podría ser lograr el acceso a indicadores desde otras plataformas como Navisworks, ya que ahora solo están disponibles en los horarios de despegue de materiales de Revit. Este enfoque del proceso de producción del edificio constituye una contribución importante a la sostenibilidad del entorno construido y la resistencia de las ciudades para mitigar el cambio climático, ya que los diseñadores mantienen el control de cada componente y sistema en tiempo real en la etapa de diseño conceptual. 


\section{AGRADECIMIENTOS}

Este trabajo es parte del proyecto de investigación "Reacondicionamiento ecoeficiente de edificios y vecindarios: gestión de residuos de construcción y demolición y la aplicación de productos ecoeficientes a la normativa española" Ref: CCPI / 2015/006, así como el Ref. Del proyecto de investigación. 3548/0632, de la Universidad de Sevilla y fue financiado por MAPEI ESPAÑA, S.A.

\section{REFERENCIAS}

Abanda, F.H., A.H. Oti, and J.H.M. Tah. 2017. "Integrating BIM and New Rules of Measurement for Embodied Energy and CO 2 Assessment." Journal of Building Engineering 12 (July): 288-305. doi:10.1016/j.jobe.2017.06.017.

"Acae Presto." 2018. Accessed January 21. http://acae.com.es//. AEC (UK). "BIM Technology Protocol v2.1." https://aecuk.files.wordpress.com/2015/ aecukbimtechnologyprotocol-v2-1-1-201506022.pdf2015.

Agencia Andaluza de la Energía. 2017. "Datos Energéticos de Andalucía 2016." Conserjería de empleo, empresa y comercio. Junta de Andalucía. https://www.agenciaandaluzadelaenergia. es/sites/default/files/documentos/datos_energeticos_de_ andalucia_2016_0.pdf.

Ajayi, Saheed O., Lukumon O. Oyedele, Boris Ceranic, Mike Gallanagh, and Kabir O. Kadiri. 2015. "Life Cycle Environmental Performance of Material Specification: A BIM-Enhanced Comparative Assessment." International Journal of Sustainable Building Technology and Urban Development 6 (1): 14-24. doi:1 0.1080/2093761X.2015.1006708.

Antón, Laura Álvarez, and Joaquín Díaz. 2014. "Integration of Life Cycle Assessment in a BIM Environment." Procedia Engineering 85: 26-32. doi:10.1016/j.proeng.2014.10.525.

Azhar, Salman, Wade A. Carlton, Darren Olsen, and Irtishad Ahmad. 2011. "Building Information Modeling for Sustainable Design and LEED ${ }^{\circledR}$ Rating Analysis." Automation in Construction 20 (2): 217-224. doi:10.1016/j.autcon.2010.09.019.

B. Delcambre. "Mission Numérique Bâtiment." http://www. territoires.gouv.fr/IMG/_ pdf/rapport_mission_numerique_ batiment.pdf2014.

Barón, J., Conde, J., Osuna, M., Ramírez, A., and Solís, J. 2017. "Consejería de Fomento y Vivienda / Vivienda y Rehabilitación / Base de Costes de La Construcción de Andalucía (BCCA) 29 Abril 2016. Banco de Precios." http://www.juntadeandalucia. es/fomentoyvivienda/portal-web/web/areas/vivienda/ texto/403b7931-0d21-11e6-a18a-052bf9b4a08b.

Basbagill, J., F. Flager, M. Lepech, and M. Fischer. 2013. "Application of Life-Cycle Assessment to Early Stage Building Design for Reduced Embodied Environmental Impacts." Building and Environment 60 (February): 81-92. doi:10.1016/j. buildenv.2012.11.009
Bey, Niki, Michael Z. Hauschild, and Tim C. McAloone. 2013. "Drivers and Barriers for Implementation of Environmental Strategies in Manufacturing Companies." CIRP Annals 62 (1): 43-46. doi:10.1016/j.cirp.2013.03.001.

"Buildings - Energy - European Commission." 2017. Energy. Accessed November 22. /energy/en/topics/energy-efficiency/ buildings.

CEN European Committe for Standardization. 2012. "UNEEN 15978. Sostenibilidad En La Construcción. Evaluación Del Comportamiento Ambiental de Los Edificios. Métodos de Cálculo." Asociación española de Normalización y Certificación (AENOR).

Chelsea. 2018. "DProfiler." Cassell Consulting - Construction Operations Consulting. Accessed August 2. https://www. cassellconsulting.com/products/dprofiler.

Chen, L., and W. Pan. 2015. "A BIM-Integrated Fuzzy Multi-Criteria Decision Making Model for Selecting Low-Carbon Building Measures." Procedia Engineering 118: 606-613. doi:10.1016/j. proeng.2015.08.490.

Cheng, Jack C.P., and Lauren Y.H. Ma. 2013. "A BIM-Based System for Demolition and Renovation Waste Estimation and Planning." Waste Management 33 (6): 1539-1551. doi:10.1016/j. wasman.2013.01.001.

Chong, Heap-Yih, Cen-Ying Lee, and Xiangyu Wang. 2017. "A Mixed Review of the Adoption of Building Information Modelling (BIM) for Sustainability." Journal of Cleaner Production 142 (January): 4114-4126. doi:10.1016/j.jclepro.2016.09.222.

De Wolf, Catherine, Francesco Pomponi, and Alice Moncaster. 2017. "Measuring Embodied Carbon Dioxide Equivalent of Buildings: A Review and Critique of Current Industry Practice." Energy and Buildings 140 (April): 68-80. doi:10.1016/j. enbuild.2017.01.075

"EQUEST." 2018. Accessed August 1. http://www.doe2.com/ equest/.

Ferreiro-Cabello, Javier, Esteban Fraile-Garcia, Eduardo Martinez de Pison Ascacibar, and Francisco Javier Martinez de Pison Ascacibar. 2016. "Minimizing Greenhouse Gas Emissions and Costs for Structures with Flat Slabs." Journal of Cleaner Production 137 (November): 922-930. doi:10.1016/j.jclepro.2016.07.153.

FIE-BDC Association. "FORMATO DE INTERCAMBIO ESTÁNDAR DE BASES DE DATOS PARA LA CONSTRUCCIÓN - FIEBDC." http://www.fiebdc.es/articulos/\#toggle-id-1.

Fiès, Bruno, Thomas Lützkendorf, and M Maria Balouktsi. 2013. "Life Cycle Sustainable Assessment and BIM." In SB13 Graz, 11. Graz.

Freire Guerrero, A., and Marrero Meléndez, M. 2015. “Evaluación a Través Del Presupuesto de La Energía Incorporada al Proyecto de Edificación." Revista Hábitat Sustentable 5 (1): 54-63.

Freire Guerrero, Antonio. 2017. "Presupuesto Ambiental. Evaluación de La Huella Ecológica Del Proyecto a Través de La Clasificación de La Base de Costes de La Construcción de Andalucía." Tesis doctoral, Seville: Universidad de Sevilla. 
Gan, Vincent J. L., Jack C. P. Cheng, Irene M. C. Lo, and C. M. Chan. 2017. "Developing a CO2-e Accounting Method for Quantification and Analysis of Embodied Carbon in High-Rise Buildings." Journal of Cleaner Production 141 (January): 825-836. doi:10.1016/j.jclepro.2016.09.126.

Gan, Vincent J.L., M. Deng, K.T. Tse, C.M. Chan, Irene M.C. Lo, and Jack C.P. Cheng. 2018. "Holistic BIM Framework for Sustainable Low Carbon Design of High-Rise Buildings." Journal of Cleaner Production 195 (September): 1091-1104. doi:10.1016/j. jclepro.2018.05.272.

González, María Jesús, and Justo García Navarro. 2006. "Assessment of the Decrease of CO2 Emissions in the Construction Field through the Selection of Materials: Practical Case Study of Three Houses of Low Environmental Impact." Building and Environment 41 (7): 902-909. doi:10.1016/j.buildenv.2005.04.006.

González-Vallejo, Patricia, Madelyn Marrero, and Jaime SolísGuzmán. 2015. "The Ecological Footprint of Dwelling Construction in Spain." Ecological Indicators 52 (May): 75-84. doi:10.1016/j. ecolind.2014.11.016.

Gul, Muhammet, Erkan Celik, Alev Taskin Gumus, and Ali Fuat Guneri. 2018. "A Fuzzy Logic Based PROMETHEE Method for Material Selection Problems." Beni-Suef University Journal of Basic and Applied Sciences 7 (1): 68-79. doi:10.1016/j. bjbas.2017.07.002.

IEA. 2013. Transition to Sustainable Buildings: Strategies and Opportunities to 2050. Paris.

Ilhan, Bahriye, and Hakan Yaman. 2016. "Green Building Assessment Tool (GBAT) for Integrated BIM-Based Design Decisions." Automation in Construction 70 (October): 26-37. doi:10.1016/j.autcon.2016.05.001.

Instituto de Tecnología de la Construcción de Cataluña. 2017. "Información Ambiental de Productos y Sistemas." Https://itec. es/. Instituto de Tecnología de La Construcción de Cataluña. Accessed June 12. https://itec.es/metabase/productossostenibles $/ \mathrm{m} / \mathrm{m} / \mathrm{m} /$.

"Introducing IESVE Software I Integrated Environmental Solutions." 2018. Accessed August 2. https://www.iesve.com/software.

Inyim, Peeraya, Joseph Rivera, and Yimin Zhu. 2015. "Integration of Building Information Modeling and Economic and Environmental Impact Analysis to Support Sustainable Building Design." Journal of Management in Engineering 31 (1): A4014002. doi:10.1061/ (ASCE)ME.1943-5479.0000308.

Jones, C., and Allen, Stephen. 2017. "Embodied Energy and Embodied Carbon." Circular Ecology. Accessed December 15. http://www.circularecology.com/embodied-energy-and-carbonfootprint-database.html.

Lamé, Guillaume, Yann Leroy, and Bernard Yannou. 2017. "Ecodesign Tools in the Construction Sector: Analyzing Usage Inadequacies with Designers' Needs." Journal of Cleaner Production 148 (April): 60-72. doi:10.1016/j.jclepro.2017.01.173.

"LEEDIUSGBC." 2018. Accessed August 9. https://new.usgbc.org/ leed?gclid=CjOKCQjwzK_bBRDDARIsAFQF7zMiWpaPRLjiG8G_ eSr_VFhH3_THFyTLyUeGIpSvfvHH2Ilo2NR7dbAaAiq8EALw_ wcB.
López-Mesa, Belinda, Ángel Pitarch, Ana Tomás, and Teresa Gallego. 2009. "Comparison of Environmental Impacts of Building Structures with in Situ Cast Floors and with Precast Concrete Floors." Building and Environment 44 (4): 699-712. doi:10.1016/j. buildenv.2008.05.017.

Lützkendorf, Thomas, Greg Foliente, Maria Balouktsi, and Aoife Houlihan Wiberg. 2015. "Net-Zero Buildings: Incorporating Embodied Impacts." Building Research \& Information 43 (1): 6281. doi:10.1080/09613218.2014.935575.

Marrero, Madelyn, and Antonio Ramirez-De-Arellano. 2010. "The Building Cost System in Andalusia: Application to Construction and Demolition Waste Management." Construction Management and Economics 28 (5): 495-507. doi:10.1080/01446191003735500.

Marrero Meléndez, M., Solís Guzmán, J., Llácer Pantión, R., Ramírez de Arellano Agudo, A., Alba Rodríguez, D., González Vallejo, P., Martínez Rocamora, A., and Ruíz Pérez, R. 2015. "GGI3003/IDIS Huella Ecológica de La Rehabilitación de Edificios: Viabilidad Económica y Ambiental. (HEREVEA)." Universidad de Sevilla. http://www.aopandalucia.es/inetfiles/resultados_IDI/ GGI3003IDIS/memoria/1_Memoria_Final_141215.pdf.

Marzouk, Mohamed, Eslam Mohammed Abdelkader, and Khalid Al-Gahtani. 2017. "Building Information Modeling-Based Model for Calculating Direct and Indirect Emissions in Construction Projects." Journal of Cleaner Production 152 (May): 351-363. doi:10.1016/j.jclepro.2017.03.138.

"Medit Mediciones en BIM con REVIT." 2013. theBIMshop.es La Tienda del BIM. http://www.thebimshop.es/bim/software-bim/ medit/.

Mercader Moyano, M. 2010. "Cuantificación de Los Recursos Consumidos y Cuantificación de Las Emisiones de CO2 Producidas En Las Construcciones de Andalucía y Sus Implicaciones En El Protocolo de Kioto." Seville: School of Architecture-University of Seville. http://fondosdigitales.us.es/tesis/tesis/1256/ cuantificacion-de-los-recursos-consumidos-y-emisiones-deco2-producidas-en-las-construcciones-de-andalucia-y-susimplicaciones-en-el-protocolo-de-kioto/.

Mercader-Moyano, Pilar, and Antonio Ramírez-de-ArellanoAgudo. 2013. "Selective Classification and Quantification Model of C\&D Waste from Material Resources Consumed in Residential Building Construction." Waste Management \& Research 31 (5): 458-474. doi:10.1177/0734242X13477719.

Ministry of Environment and Rural and Marine Affairs. 2008. II Plan Nacional de Residuos de Construcción y Demolición Para El Período 2008-2015. Boletín Oficial del Estado. Madrid: Spanish Government.

Mousa, Michael, Xiaowei Luo, and Brenda McCabe. 2016. "Utilizing BIM and Carbon Estimating Methods for Meaningful Data Representation." Procedia Engineering 145: 1242-1249. doi:10.1016/j.proeng.2016.04.160.

Najjar, Mohammad, Karoline Figueiredo, Mariana Palumbo, and Assed Haddad. 2017. "Integration of BIM and LCA: Evaluating the Environmental Impacts of Building Materials at an Early Stage of Designing a Typical Office Building." Journal of Building Engineering 14 (November): 115-126. doi:10.1016/j. jobe.2017.10.005. 
Official Journal of the European Communities., and Commission Decision 2001/118/EC of wastes. 2001. "European Waste Catalogue."

Official Journal of the European Union. 2010. EPBD Recast: Directive 2010/31/EU on the Energy Performance of Buildings.

Official Journal of the European Union. 2012. "Guidelines Accompanying Commission Delegated Regulation (EU) No 244/2012 of 16 January 2012 Supplementing Directive 2010/31/ EU of the European Parliament and of the Council on the Energy Performance of Buildings by Establishing a Comparative Methodology Framework for Calculating Cost-Optimal Levels of Minimum Energy Performance Requirements for Buildings and Building Elements."

Ramírez de Arellano Agudo, Antonio. 2002. Retirada selectiva de residuos: modelo de presupuestación. Sevilla: Colegio Oficial de Aparejadores y Arquitectos Técnicos de Sevilla.

Rodríguez Serrano, Antonio, and Santiago Porras Álvarez. 2016. "Life Cycle Assessment in Building: A Case Study on the Energy and Emissions Impact Related to the Choice of Housing Typologies and Construction Process in Spain." Sustainability 8 (3): 287. doi:10.3390/su8030287.

Sandberg, Nina Holck, and Helge Brattebø. 2012. "Analysis of Energy and Carbon Flows in the Future Norwegian Dwelling Stock." Building Research \& Information 40 (2): 123-139. doi:10. 1080/09613218.2012.655071.

Solís Guzmán, J. 2010. "Evaluación de La Huella Ecológica Del Sector Edificación (Uso Residencial) En La Comunidad Andaluza." Doctoral thesis, Seville: University of Seville.

Soust-Verdaguer, Bernardette, Carmen Llatas, and Antonio García-Martínez. 2017. "Critical Review of Bim-Based LCA Method to Buildings." Energy and Buildings 136 (February): 110120. doi:10.1016/j.enbuild.2016.12.009.

"Spain Launches BIM Strategy with Pencilled-in 2018 Mandate | BIM+." 2018. Accessed February 3. http://www.bimplus.co.uk/ news/spain-launches-bim-strategy-pencilled-2018-mandate/.

Spanish Government - Ministry of the Presidency. 2008. "Real Decreto 105/2008, de 1 de Febrero, Por El Que Se Regula La Producción y Gestión de Los Residuos de Construcción y Demolición (National Decree 105/2008, February 1, Which Regulates the Production and Management of Construction and Demolition Waste)." Ministry of the Presidency. Madrid. Spain.

Surveyors, Royal Institution of Chartered. 2018. "RICS NRM: New Rules of Measurement." Accessed August 1. http://www.rics.org/ uk/knowledge/professional-guidance/guidance-notes/new-rulesof-measurement-order-of-cost-estimating-and-elemental-costplanning/.

Swiss Centre for Life Cycle, and Inventories. 2017. "Ecoinvent Version 3." Accessed December 15. http://www.ecoinvent.org/ database/database.html.

"Tally." 2018. Accessed August 1. http://www.choosetally.com/.

Tiwari, Piyush. 2001. "Energy Efficiency and Building Construction in India." Building and Environment 36 (10): 1127-1135. doi:10.1016/S0360-1323(00)00056-1.
"United Nations Framework Convention on Climate Change." 2018. Accessed January 21. http://unfccc.int/2860.php.

Villoria Sáez, Paola, Jaime Santa Cruz Astorqui, Mercedes del Río Merino, María del Pilar Mercader Moyano, and Antonio Rodríguez Sánchez. 2018. "Estimation of Construction and Demolition Waste in Building Energy Efficiency Retrofitting Works of the Vertical Envelope." Journal of Cleaner Production 172 (January): 2978-2985. doi:10.1016/j.jclepro.2017.11.113.

Wong, Johnny Kwok Wai, and Jason Zhou. 2015. "Enhancing Environmental Sustainability over Building Life Cycles through Green BIM: A Review." Automation in Construction 57 (September): 156-165. doi:10.1016/j.autcon.2015.06.003.

Yang, Xining, Mingming Hu, Jiangbo Wu, and Bin Zhao. 2018. "Building-Information-Modeling Enabled Life Cycle Assessment, a Case Study on Carbon Footprint Accounting for a Residential Building in China." Journal of Cleaner Production 183 (May): 729-743. doi:10.1016/j.jclepro.2018.02.070.

Yeheyis, Muluken, Kasun Hewage, M. Shahria Alam, Cigdem Eskicioglu, and Rehan Sadiq. 2013. "An Overview of Construction and Demolition Waste Management in Canada: A Lifecycle Analysis Approach to Sustainability." Clean Technologies and Environmental Policy 15 (1): 81-91. doi:10.1007/s10098-0120481-6.

ZukunftBAU. 2013. "A Research Program of the Federal Ministry for Transport, Building and Urban Affairs (BMVBS)." Federal Republic of Germany. http://www.aec3.com/ de/downloads/BIMGuide-Germany.pdf). 